\title{
Implementation of Modular Algebraic Specifications
}

\author{
(extended abstract) \\ N.W.P. van Diepen \\ Centre for Mathematics and Computer Science \\ P.O. Box 4079, 1009 AB Amsterdam, The Natherlands
}

\begin{abstract}
The foundation of implementation of algebraic specifications in a modular way is investigated. Given an algebraic specification with visible and hidden signature an observing signature is defined. This is a part of the visible signature which is used to observe the behaviour of the implementation.

Two correctness criteria are given for the implementation with respect to the observing signature. An algebraic correctness criterion guarantees initial algebraic semantics for the specification as seen through the observing signature, while allowing freedom for other parts of the signature, to the extent that even final semantics may be used there. A functional correctness criterion allows one to prove the correctness of the implementation for one observing function in Hoare logic. The union over all observing functions of such implementations provides an actual implementation in any programming language with semantics as described above.

Note: Partial support has been received from the European Communities under ESPRIT project no. 348 (Gen* eration of Interactive Programming Environments - GIPE).
\end{abstract}

\section{INTRODUCTION}

An algebraic specification is a mathematical structure consisting of sorts, functions (and constants) over these sorts, and equations describing the relation between the functions and constants. It is a convenient tool to specify static and dynamic semantics of programming languages, see e.g. Goguen and Meseguer ([GM82], [GM84], [MG85]) for more detail on algebraic specification, and [BHK85], [BDMW81] and [Die86] for examples. The implementation of an algebraic specification usually consists of the conversion of the equations into a term rewriting system, either directly or through the completion procedure of Knuth-Bendix. More detail can be found in [HO80] and [ODO85]. The performance of such an implementation is rather slow in general, compared with algorithms written in conventional programming languages, while the specification must have certain properties to be implemented in this way at all. The aim of this paper is to provide another implementation strategy, based on pre- and postconditions, allowing the application of more classical programming and optimization techniques.

\subsection{Modular algebraic specifications}

Algebraic specifications have been introduced to provide a description style for data types in an algebraically - mathematically - nice way. The mathematical notion of a (many-sorted) algebra used here is a structure consisting of carrier sets and typed functions (including constants) over these sets, together with a set of equations, specifying the behaviour of the functions. The combination of a set of sorts (the names of the carrier sets) and a set of functions (which include constants, unless stated otherwise), is called the signature of the algebra.

The algebraic specifications studied in this paper have additional organization primitives, prompted by both theoretical and practical considerations. Ceniral issue is the modular structure imposed on the algebraic specifications. An aigebraic specification can import another algebraic specification as a module, meaning that it adds the sorts, functions and equations of the imported specification to its own. Sorts or functions with the same name are only allowed when they are the same (they must originate in the same module), otherwise they must be renamed.

The modular approach naturally leads to two other primitives, a parameter mechanism, and the occurrence of hidden (local, auxiliary) sorts and functions. Hidden sorts and functions (hiddens) are used in the equations of the module in which they are defined, but they are not included in the exported or visible sorts and functions. Only the latter are included in the algebra associated with the module. Hiddens make it easier to write many specifications by providing local definitions, and they are necessary to specify properties needing an infinite number of equations (when defined without hiddens) in a finite way ([BT83], [BT82]).

The equations used are conditional equations, i.e. equations which are valid only when certain conditions are satisfied. The semantics provided are of an initical nature, which will be explained and modified in the paper. These semantics are usually intuitively clear. 


\subsection{Implementation of algebraic specifications}

Once an algebraic specification has been written there is no clearcut way to derive a working program from it. In general, any model of the algebra can be seen as an implementation. Certain models are preferable, though.

A strategy followed quite often to implement a model satisfying initial semantics (an initial model) is to transforn the specification into a term rewriting system. The easiest way to do this is to give every equation a direction, say from left to right, and to view the set of directed equations as a set of rewrite rules, transforming one term over the signature into another. This system can be found in various places in the literature ([BK86], [DE84], [FGJM85], [GMP83], [ODo85], [Die86]), but the success of this method depends on the properties of the directed version of the (in principle undirected) set of equations, combined with the technique used for rewriting. Tuming an equation around (writing $B=A$ instead of $A=B$ ) or writing the equations in a different order may have significant consequences for the behaviour (both in speed and in termination) of the implementation, while the specification has not been changed, except textually.

An additional problem is what to do with the modular structure when fitting a modular specification in a term rewriting system. Transparent semantics can be obtained by a normalization step (as described by Bergstra, Heering and Klint [BHK86]), flattening all imports into one module (renaming hidden functions and sorts where necessary). The approach above can be applied to the normalized module. It may be debated whether the loss of the structure in the specification is sufficiently motivated by the transparency of the semantics.

The present paper aims at a more module-oriented implementation, giving semantics to implement an observing signature (a signature through which one can observe the visible signature, of which it is a subsignature) in a functional way, using descriptions of the observing functions in Hoare logic (see e.g. the text books [LS84], [Bac86]). The significance for the semantics of the import construct will be touched upon briefly. The main advantage of this approach is that it permits the implementation of modules in a, possibly low-level, efficient way from the high-level specification. This allows the construction of a library of efficient basic modules upon which more sophisticated algebraic specifications may depend.

\subsection{Related work}

Implementation techniques for pure initial semantics are burdened with the obligation to implement the initial algebra semantics faithfully. This generally slows down the implementation, since often an initial specification demands too much detail, as has been discussed by Baker-Finch [Bak84].

In [MG85] and [ST85] another implementation criterion for algebraic specifications is provided. They focus on observable sorts, while the present paper takes a functionaily oriented point of view. Their approach is a special case of the approach presented here. More detail can be found in section 3.2.2.

There is a strong resemblance to abstract data type theory as practiced in the verification of correctness of programs (cf. Jones [Jon80]). After all, an algebraic specification is a nice way to describe a data type. While the specifications look similar, the point of view is different. Constructor functions (i.e. functions describing the data type) really construct the type in algebraic specifications, while they only serve as a description tool in [Jon80].

Techniques which use term rewriting systems have the advantage of allowing (semi-)automatic translation schemes, but pay the price with severe restrictions on the set of equations allowed. Perhaps the overhead of a completion procedure for generation of rewrite nules is needed, e.g. the Knuth-Bendix procedure (see [HO80] and [OD085] for more detail). The technique presented here allows for faster implementations, but does not support automatic translation.

\subsection{An outline of this paper}

In section 2 brief introductions to both specification formalisms used (the algebraic specification formalism ASF and Hoare logic) are given. Section 3 begins with a description of certain disadvantages of the initial algebra approach to motivate the theoretical framework leading to an algebraic implementation notion in the second half. An example giving an implementation according to this notion follows in section 4 , which may be read before section 3.2 to get the flavour, or in the order provided to convince oneself of the rigour of the approach. The functional implementation notion is described as an extension of the algebraic notion in section 5, preceded by an example to show the insufficient strength of the latter notion for our purpose. The example of section 4 is implemented in an imperative language in section 6 according to this notion. Finally some conclusions are formulated in section 7.

\section{THE FORMALISMS INFORMALLY}

\subsection{Algebraic specifications}

The algebraic specifications in this paper are presented in the specification formalism ASF (for Algebraic Specification Formalism), of which a complete treatment of syntax and semantics is given by Bergstra, Heering and Klint in [BHK87]. The 
choice of ASF is not essential to the paper, so there is no need to explain this formalism in great detail. Various features (e.g., renaming, infix operators) are not used in the paper and will not be discussed here at all.

A specification in ASF consists of a list of modules. Every module is named and contains the following (optional) sections:

- A parameters section, which contains a list of sorts and functions to be bound to fully define the module.

- An exports section, containing the sorts and (typed) functions visible to the outside world (which is an importing module).

- An imports section, containing a list of modules. The sorts and functions exported by the modules in the list are imported in the current list and exported again. Parameters can be bound in this section. The sorts and functions of this and the preceding section provide the visible signature in the corresponding formal treatment of the algebraic specification.

- A sorts section, and a functions section, providing the hidden signature of the algebraic specification. These two sections together are often informally dubbed the hidden section. Definitions here are local to the module.

- A variables section, which quantifies the list of typed variables universally over the equations presented in the

- equations section, containing positive conditional equations (i.e., equations of the form $A=B$ or $A=B$ when $P_{1}=Q_{1} \wedge \cdots \wedge P_{n}=Q_{n}$ ). Terms in equations consist of open terms generated by all (also the hidden) functions.

The equations which hold in a module are all equations of the module itself and the equations of the (directly and indirectly) imported modules with proper renaming of hidden functions, sorts and variables from the imported modules.

A special place in ASF has the function if, which is predefined. This function has three arguments of which the first must be of type BOOL and the second and third of the same, but arbitrary, type. The term if (bool, $x, y$ ) (with variables $x, y$ of the same sort) may be interpreted as if bool then $x$ else $y$.

The semantics of a module is defined by the initial algebra over the export (visible) signature and the function if. This will be encountered in more detail in the remainder of the paper. Various ASF-specifications will appear in the sequel, to which one is referred for examples.

\subsection{Hoare logic and abstract data types}

Hoare logic is a well-known technique to describe the behaviour of programs in both imperative and functional languages. It has found its way into various text books, e.g. [LS84] and [Bac86]. Briefly, Hoare logic allows one to write [P] $S$ [Q], meaning that evaluation of program $S$ in a state in which precondition $P$ holds results in a state in which postcondition $Q$ holds. These conditions describe the state vector, i.e., the variables and their contents, of the program. Various proof rules and proof techniques are available to verify such a program.

In the paper some functions specified in an algebraic way will be specified in an equivalent Hoare logic way by giving conditions on its input terms and its output. Such a specification is independent of the actual implementation program, which may be changed (and preferably optimized). Since Hoare logic techniques can be formulated for many languages the ultimate program could be written in any appropriate language, at a cost in interfacing. Hence a large degree of language independence for the implementation is achieved, allowing various kinds of optimization strategies.

One way to interpret an algebraic specification is as a high level specification of an abstract data type. Hence the implementation strategy for algebraic specifications presented here bears a more than castal resemblance to the theory of implementation of abstract data types. An abstract data type is some type together with a set of functions on the type. An implementation is a more concrete (i.e., closer to machine level) type with a corresponding set of functions which model the abstract type and functions. This is done by providing a translation back and forth between the abstract and concrete types, such that the abstract functions are simulated correctly by the combination of the translation to the concrete type, application of the concrete function and the translation back to the abstract type (cf. Jones [Jon80]).

The scheme in the paper basically relaxes the translation conditions for all terms in the initial model of the algebraic specification by demanding translations for specific terms only. This stems from the functional orientation: only the input terms need to be translated and only the output terms need to be translated back. Section 5 provides more detail.

\section{ALGEBRAIC IMPLEMENTATION}

\subsection{Initial algebra semantics and rewsability}

The question we want to consider is the following. Suppose we have an algebraic specification and we want to provide an efficient implementation for further use by someone else, as in a library. What is the interaction between efficiency and sernantics?

Initial algebra semantics have much in favour. They are characterized by 'no junk', i.e., it is clear which objects exist, and "no confusion', i.e., closed terms (terms without variables) are only equal if they can be proved equal using equational logic. While these two characterizations are clearly desirable in many circumstances this is not always the case.

The 'no confusion' condition generates overspecification in the sense that terms might be distinguished from each other without necessity. If the writer of a specification does not care about whether two terms are equal or not (in the common case 
that their usage is identical), and hence does not specify their equality, they are unequal. This puts a burden on the implementor of the specification to provide this inequality, not allowing a possibly more efficient identification. If such an identification could be allowed the burden is shifted to the shoulders of the specifier, who has to provide additional equations. While the extra amount of work is undesirable it is also not clear in general what additional equations are necessary and whether a sufficient set can be found at all. For discussions see [Bak84], [Kam83] and [Wan79].

Apart from the question whether a satisfactory solution can be found in general this is contrary to the amount of detail one wants to specify algebraically. For this two important considerations can be given, one philosophical and one practical:

- Algebraic specification is a higher level programming formalism. While the formalism is powerful enough to express bit level if necessary, this is a waste of effort. There are more than enough lower level programming languages already.

- An algebraic specification (and indeed any specification) is made with a certain use in mind. This use is what has to be specified in detail, since that is what has to be implemented. Other details specified are peripheral in the sense that one might have chosen another description. The less detail is fixed by these peripheral specifications the more freedom an implementor has for optimizing it. The choice of models for implementation should not be restricted to one model (up to isomorphism), but rather be as broad as the class of all models. So demanding the implementation of initial algebra semantics is too restrictive.

For the remainder of the paper we distinguish three important subsets in the signature of an algebraic specification. The consequences of this tripartition for the theory are investigated in the next section. These subsets are:

- The visible signature which generates the terns existing in the specification for the outside world.

- The hidden signature, which is necessary to obtain finite initial algebra specifications on the one hand and useful for alternative data description on the other hand. The complete signature is the union of the visible and the hidden signature.

- The observing signature, which restricts the terms generated by the visible signature. It contains the functions through which visible terms may be used and the sorts with terms which may be used as observing terms. A term is an observing term if both the head function and its sort are in the observing signature. This signature is the subset of the visible signature, that one wants to be implemented as specified.

The choice of the functions and sorts in the observing signature depends on the goal one has in mind for the specification. Making this signature bigger enhances the possibilities for use but restricts the freedom of the implementor. So one can opt for a fast, but narrowly applicable implementation, or for a more generally usable, but slower implementation.

Of course, the speed of a certain function in an observable signature is not only dependent on the signature but also on the implementations of other (not necessarily observing) functions. One can for instance trade the speed of an insertion function for the speed of a retrieval function by gearing the underlying data structure (at this level of abstraction represented by the visible functions that are not observing and the hidden functions) to the other task.

\subsection{A theory of algebraic implementation}

This section is devoted to the development of a theory for the subseguently introduced notion of algebraic implementation with respect to an observing signature. Roughly speaking two algebraic specifications are algebraic implementations of each other when the behaviour of the observing functions is the same in both specifications. An annotated example is provided in section 4 . The reader may wish to read the example first, referring back to notations and details in this section where needed.

\subsubsection{Notations (algebraic specifications)}

In the rest of the paper the following conventions are used:

a. A signature $\Sigma$ is a cuple $(S, F)$ in which $S$ is a set of sorts and $F$ a set of typed functions. (Note that there is no intrinsic relation between the sorts in $S$ and $F$.) Often an element of $F$ is denoted by its name only, providing typing when necessary. Two functions with the same name, but different typing are different functions.

b. A complete signature $\Sigma=(S, F)$ is a signature in which for all $f: s_{1} \times \cdots \times s_{k} \rightarrow s \in F$ holds that all sorts in the typing of $f$ are available in $\mathrm{S}$, so $s_{1}, \cdots, s_{k}, s \in \mathrm{S}$.

c. For a signature $\Sigma$ is $T(\Sigma)$ the set of closed terms; $T_{s}(\Sigma)$ is the subset of terms of sort $s$ from $T(\Sigma)$.

d. Union, intersection and inclusion are defined for signatures $\Sigma_{1}, \Sigma_{2}\left(\Sigma_{i}=\left(S_{i}, F_{i}\right)\right)$, as:

$$
\Sigma_{1} \cup \Sigma_{2}=\left(S_{1} \cup S_{2}, F_{1} \cup F_{2}\right), \quad \Sigma_{1} \cap \Sigma_{2}=\left(S_{1} \cap S_{2}, F_{1} \cap F_{2}\right) \text {, and } \Sigma_{1} \subseteq \Sigma_{2}=S_{1} \subseteq S_{2} \wedge F_{1} \subseteq F_{2} \text {. }
$$

e. An algebraic specification is a tuple $\left(\Sigma_{V}, \Sigma_{H}, \mathrm{E}\right)$ with

- $\Sigma_{V}=\left(S_{V}, F_{V}\right)$ a complete signature (the visible signature),

- $\Sigma_{H}=\left(S_{H}, F_{H}\right)$ a signature (the hidden signature) such that $\Sigma_{V} \cup \Sigma_{H}$ (the internal signature) is a complete signarure, and

- E a set of equations over $T\left(\Sigma_{W} \cup \Sigma_{H}\right)$.

f. Let $\left(\Sigma_{V}, \Sigma_{H}, \mathrm{E}\right)$ be an algebraic specification and let $t, t^{\prime} \in T\left(\Sigma_{V} \cup \Sigma_{H}\right)$. For an equation $e \in E$, $t$ and $t^{\prime}$ are equal through direct substitution in equation $e$ (i.e., in one step) is written as $t={ }_{e} t$. 
$t$ and $t^{\prime}$ are equationally equal, i.e., equal through zero or more direct substitutions in one or more equations from $\mathrm{E}$, is written as $t=\mathrm{E} t$.

3.2.2. Definitions ( $\Sigma_{O}$-observability and -equality)

Let $\left(\Sigma_{V}, \Sigma_{H}, E\right)$ be an algebraic specification and $\Sigma_{O}=\left(S_{O}, F_{O}\right)$ (the observing signature) a signature such that $\Sigma_{O} \subseteq \Sigma_{V}$.

a. The set of closed $\Sigma_{o}$-terms over $\Sigma_{V}$, also called the set of observing terms is the set of terms in $T\left(\Sigma_{V}\right)$ of sort in $S_{O}$ and head function symbol in $\mathrm{F}_{O}$. It is defined as:

$$
T\left(\Sigma_{O}, \Sigma_{V}\right)=\left\{t \in T\left(\Sigma_{V}\right) \mid \exists f: s_{1} \times \cdots \times s_{k} \rightarrow s \in \mathbf{F}_{O}, s \in \mathrm{S}_{O} \exists u_{1} \in T\left(\Sigma_{V}\right) \cdots \exists u_{k} \in T\left(\Sigma_{V}\right)\left\{t \in T_{s}\left(\Sigma_{V}\right) \wedge t=f\left(u_{1}, \cdots, u_{k}\right)\right]\right\} .
$$

The set of closed $\Sigma_{O}$-terms over $\Sigma_{V}$ of sort $s$ is written as $T_{s}\left(\Sigma_{O}, \Sigma_{V}\right)$.

Note that it is possible to have functions in $\mathrm{F}_{O}$ whose output sort is not in in $\mathrm{S}_{O}$, or sorts in $\mathrm{S}_{O}$ which cannot be reached from $F_{o}$. This choice of notation is motivated by the function-oriented approach of this paper. By choosing a set of observing functions $F_{O}$ and all visible sorts $S_{V}$ for $S_{O}$ all sorts in $S_{V}$ which cannot be reached do not influence $T\left(\Sigma_{O}, \Sigma_{V}\right)$. For symmetry reasons the definition is formulated in such a way that one can also restrict the sorts and not the functions, as will be done in point $e$ below. Alternatively, it is possible to define $S_{O}$ as the set of sorts in the range of $F_{O}$ without affecting the theory.

b. Where no confusion can arise the abbreviations $T_{O}=T\left(\Sigma_{O}, \Sigma_{V}\right), T_{V}=T\left(\Sigma_{V}\right), T_{s, o}=T_{s}\left(\Sigma_{O}, \Sigma_{V}\right)$, and $T_{s, V}=T_{s}\left(\Sigma_{V}\right)$ are used.

c. A context (for sort $s$ ) $\mathrm{T}(g)$ is a term with a missing subterm of sort $s$. The empty context (i.e., a context in which the top term is missing) is written as a.

A term $t \in T_{s, V}$ is $\Sigma_{O}$-observable if and only if there exists a context $\mathrm{T}\left(\varphi_{s}\right)$ such that $\mathrm{T}(t) \in T_{O}$;

a $\Sigma_{O}$-observable term $t \in T_{s, V}$ is directly $\Sigma_{O}$-observable if and only if $t \in T_{O}$ (the empty context $T\left(\varphi_{s}\right)=$ satisfies $T(t) \in T_{O}$ ); a $\Sigma_{o}$-observable term $t \in T_{s_{1} V}$ is indirectly $\Sigma_{O}$-observable if and only if $t \in T_{O}\left(T\left(\varphi_{s}\right)=\bullet_{s}\right.$ does not satisfy $\left.T(t) \in T_{O}\right)$.

d. $\Sigma_{0}$ equality (i.e., equality with respect to observations through terms in the observing signature $\Sigma_{0}$ ) is defined as follows for two terms $t, t^{\prime} \in T_{s, V}$ :

$$
t-\mathrm{E}, \Sigma_{0} t^{\prime} \Leftrightarrow \forall \mathrm{T}\left(\varphi_{s}\right)\left[\mathrm{T}(t), \mathrm{T}\left(t^{\prime}\right) \in T_{O} \rightarrow \mathrm{T}(t)=\mathrm{E} \mathrm{T}\left(t^{\prime}\right)\right] .
$$

Where no confusion can arise $\sim E, \Sigma_{0}$ is abbreviated to $-O$.

e. Let $f \in \mathrm{F}_{Y}$. A term $t \in T\left(\Sigma_{V}\right)$ is $f$-observable if and only if $t$ is $\left(\mathrm{S}_{V},\{f\}\right)$-observable; two terms in $T\left(\Sigma_{V}\right)$ are $f$-equal if and only if they are $\left(S_{V},(f)\right)$-equal.

Let $s \in S_{V}$. A term $t \in T\left(\Sigma_{V}\right)$ is $s$-observable if and only if $t$ is $\left(\{s\}, \mathbf{F}_{V}\right)$-observable; two terms in $T\left(\Sigma_{V}\right)$ are $s$-equal if and only if they are $\left(\{s\}, F_{V}\right)$-equal.

Since the definition in case $c$ ignores unreachable sorts and functions to unavailable sorts, only terms with head symbol $f$ in the first two cases, and with range $s$ in the last two, are relevant.

The notion of observability via a sort corresponds to the notion in [MG85], and is underlying the behavioural equivalence notion in [ST85]. In the latter paper, the observational equivalence notion is very general, since it is parameterized with the logic used to reason about observations. Thus $\Sigma_{o}$-equality corresponds to observational equivalence under conditional equational logic in the terms of [ST85]. By concentrating here on one logic more can be said about the implementation.

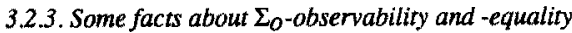

In final algebra semantics terms are equal unless they can be proved different, so in models with final semantics there is the maximum amount of 'confusion' consistent with the inequalities which must exist in the model. As such, $\Sigma_{O}$-equality is a notion from final algebra semantics. If you want to show that two closed terms are different you have to find a context for which these terms behave differently, thus proving their inequality. If no such context can be found the terms cannot be distinguished from each other. In initial semantics, on the contrary, they are distinguished unless they are equationally equal, in other words, unless they can be transformed into each other via equations from $E$, thus proving their equality.

Let $\left(\Sigma_{V}, \Sigma_{H}, E\right)$ be an algebraic specification, $\Sigma_{O} \subseteq \Sigma_{V}$, and $t, t^{\prime} \in T_{s, V}$, then the following facts hold:

a. $T\left(\Sigma_{v}, \Sigma_{V}\right)=T\left(\Sigma_{V}\right)$.

Observing through the visible signature gives all visible terms. This follows immediately from definition 3.2.2.a.

b. If $\Sigma_{o}^{\prime} \subseteq \Sigma_{o}$ then $T\left(\Sigma_{o}^{\prime}, \Sigma_{\gamma}\right) \subseteq T\left(\Sigma_{0}, \Sigma_{y}\right)$.

A smaller observing signature results in less observing terms. Again this follows from definition 3.2.2.a.

c. If $\Sigma_{o}^{\prime} \subseteq \Sigma_{o}$ then $t-{\mathrm{E}, \Sigma_{o}}^{\prime} \rightarrow t-\mathrm{E}, \Sigma_{o} t^{\prime}$.

This follows from the definition of $\Sigma_{O}$-equality and fact $b$, since there are less contexts in $T\left(\Sigma^{\prime}{ }_{O}, \Sigma_{V}\right)$ than in $T\left(\Sigma_{O}, \Sigma_{V}\right)$ to show the difference between $t$ and $t^{\prime}$.

d. If $t$ and $t$ are not $\Sigma_{O}$-observable they are $\Sigma_{O}$-equal. 
Since there is no context to show the difference between $t$ and $t^{\prime}$ this follows from the definition.

e. If $t$ is $\Sigma_{O}$-observable and $t^{\prime}$ is not then they are $\Sigma_{O}$-equal.

The argument for fact $d$ holds here also.

f. It should be noted that in cases $d$ and $e$ both $t \sim E, \Sigma_{r} t^{\prime}$ and $\neg t-E, \Sigma_{r} t^{\prime}$ can be true. Take for example:

$$
\begin{aligned}
& \Sigma_{V}=([s],\{a, b]) \text { with } a, b \in s, \text { and } \\
& \Sigma_{O}=(\{s\},\{a]) \text { for case e, or } \Sigma_{O}=([s\}, \varnothing) \text { for case d. }
\end{aligned}
$$

When $\mathrm{E}$ is empty $a-\varnothing, \Sigma_{\phi} b$ and $\neg a-\varnothing, \Sigma_{\nu} b$ hold; $\mathrm{E}=\{a=b\}$ has as result that $a \sim\{a=b], \Sigma_{0} b$ and $a-\{a=b], \Sigma_{\nu} b$.

The following lemma states that the initial algebraic structure is retained on directly observable terms. So only indirectly observable and unobservable terms can lose their initial behaviour. It follows immediately (corollary 3.2 .5 ) that no restriction on the observability (i.e., $\Sigma_{Q}=\Sigma_{V}$ ) retains the initial algebraic structure.

\subsubsection{Intial Algebra Lemma}

For $t, t^{\prime} \in T_{s}\left(\Sigma_{O}, \Sigma_{V}\right): t-\mathrm{E}, \Sigma_{0} t^{\prime} \Leftrightarrow t=\mathrm{E} t^{\prime}$.

Proof direct from definition 3.2.2.d (omitted).

\subsubsection{Corollary $\left(\Sigma_{O}=\Sigma_{V}\right.$ preserves initial algebra semantics)}

For $t_{2} t^{\prime} \in T_{s}\left(\Sigma_{V}\right): t-\mathrm{E}, \Sigma_{2} t^{\prime} \Leftrightarrow t=\mathrm{E} t^{\prime}$.

\subsubsection{Witness Existence Lemma}

The following lemma formulates a nice fact for proofs with observable terms. Two terms are $\Sigma_{0}$-equal unless there is a context proving the opposite. Hence two terms are $\Sigma_{O}$-equal when there is no common context. So it is important to have at least one common context. In this lemma existence of a witness context is asserted for $\Sigma_{O}$-observable terms of the same sort.

Lemma: For two $\Sigma_{O}$-observable terms $t, t^{*} \in T_{s, V}$ there exists a context $\mathrm{T}\left(\boldsymbol{o}_{s}\right)$ such that $\mathrm{T}(t), \mathrm{T}\left(t^{\prime}\right) \in T_{O}$.

Proof (omitted).

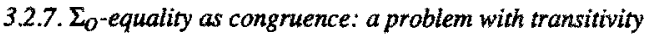

We would have liked to use the notation of $=o$ instead of $-o$ since it should define a congruence similar to $=\mathrm{E}$. However, there are some problems connected with the final nature of $\sim_{O}$ and the initial nature of $=_{E}$. A congruence - satisfies the laws of symmetry $(t-t)$, reflexivity (if $t-t^{\prime}$ then also $t^{\prime}-t$ ), transitivity (if $t-t^{\prime}$ and $t^{\prime}-t^{\prime \prime}$, then also $t-t^{\prime \prime}$ ), and the substitution property, (if $t_{1}-t_{1}{ }^{\prime} \wedge \cdots \wedge t_{n}-t_{n}{ }^{\prime}$ holds then also $f\left(t_{1}, \cdots, t_{n}\right) \sim f\left(t_{1}{ }^{\prime}, \cdots, t_{n}{ }^{3}\right.$ holds). For terms in $T\left(\Sigma_{V}\right)$ reflexivity, symmetry and the substitution property of $\sim o$ follow immediately from the corresponding properties of $=_{\mathrm{E}}$ and definition 3.2.2.d. However, in section 3.2.3 facts $\mathrm{d}$ and e show that transitivity is not guaranteed on $T\left(\Sigma_{V}\right)$. Since these facts deal with terms that are not observable, this is no real problem. However, $\sim_{O}$ is also not transitive on the subset of $\Sigma_{o}$-observable terms in $T\left(\Sigma_{v}\right)$. This is illustrated in the following example.

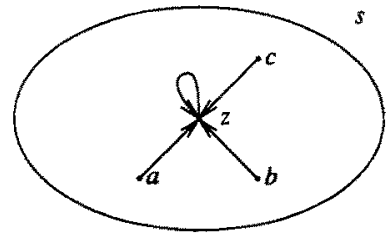

FIGURE 3.1

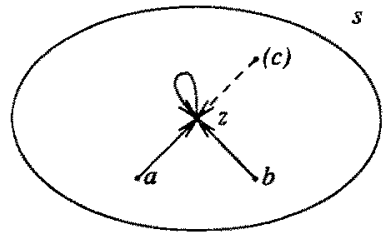

FIGURE 3.2

Let $\Sigma_{V}=(\{s\},\{a, b, c, z, f])$ with $a, b, c, z \in s$ and $f: s \rightarrow s$, E consists of the equation $f(x)=z$ with $x$ a variable of sort $s$, and $\Sigma_{o}=(\{s\},\{a, b, z, f\})$. This structure is shown in FIGURE 3.1. Then $a \sim o c$, since $f(a)=_{\mathrm{E}} f(c), f(f(a))==_{\mathrm{E}} f(f(c))$, etc., and similarly $c \sim o b$, though $\neg a \sim_{o} b$. Still, this is not unreasonable. If one only looks at $\Sigma_{0}$ any relation involving $c$ is irrelevant. The new structure is given in FIGURE 3.2, forgetting the dashed arrow. If later on one would want to add a new constant named $c$, then $c$ could be a new name for an old constant like $a, b$, or $z$, or a completely new constant. So if $c$ is observably not equal to one or more of the constants, this would rule out some of the possibilities. Hence, the freedom allowed when introducing $c$ would be limited in an undesirable way.

The precise criteria conserving transitivity, and hence making -o a congruence are given in Theorem 3.2.8. Some important classes of observable signatures that are transitivity conserving are given in a corollary $(3.2 .9)$. 


\subsubsection{Transitivity Theorem}

Let $t, t^{\prime}, t^{\prime \prime}$ be $\Sigma_{O}$-observable terms of sort $s$ such that $t$ - $_{0} t^{\prime}$ and $t^{\prime \prime}-_{O} t^{\prime \prime}$, then

$$
\neg t \sim o t^{\prime \prime} \Leftrightarrow t, t^{\prime \prime} \in T_{O} \wedge t^{\prime} \in T_{V}-T_{O} \wedge \forall \mathrm{T}\left(\bullet_{s}\right) \mathrm{T}(t), \mathrm{T}\left(t^{\prime \prime}\right) \in T_{O} \rightarrow\left[\mathrm{T}(t) \neq_{\mathrm{E}} \mathrm{T}\left(t^{\prime \prime}\right) \leftrightarrow \mathrm{T}\left(\bullet_{s}\right)=\bullet_{s}\right]
$$

Proof based on the fact that ${ }_{s}$ is used to compare $t$ with $t^{\prime \prime}$, but not $t^{\prime}$ with $t$ or $t^{\prime \prime}$ (omitted).

\subsubsection{Transitivity conserving constraints}

The transitivity theorem states that two directly $\Sigma_{O}$-observable terms can be $\Sigma_{O}$-unequal, even though there is an indirectly $\Sigma_{0}$-observable term which is $\Sigma_{O}$-equal to both. This is the case in the example in 3.2 .7 . Hence $\Sigma_{O}$-inequality is stronger for directly observable terms than for indirectly observable terms.

The theorem above provides necessary and sufficient conditions for transitivity. This may be unwieldy to use in practice. However, it is conveniently possible to give criteria, that are important from the point of view of implementation, to check whether $-a$ is an equivalence relation. Intuitively, the implementation of directly observable terms only has to follow the initial algebra semantics (Lemma 3.2.4), while indirectly observable and unobservable terms are less demanding for the implementation. Some criteria are formulated below:

Corollary:

Let $T_{s}$ be the subset of $\Sigma_{O}$-observable terms from $T_{s,}$. . The relation $\sim_{\mathrm{E}, \Sigma_{\mathrm{o}}}$ is an equivalence relation on $T_{s}$ if either of the following holds:

a. $T_{s, 0}=\varnothing$, i.e., sort $s$ is not directly observable. Consequently its internal representation may be changed without altering the directly observable sorts.

b. $T_{s, O}=T_{s}$, i.e., $T_{s}$ has to be implemented with initial algebra semantics.

c. for all $t \in T_{s}$ there is precisely one $t^{\prime} \in T_{s, O}$ such that $t^{\prime} \sim_{O} t$, i.e, there is exactly one directly observable term $\Sigma_{O}$-equal to any term of $T_{s}$. This term plays the role of a canonical form and has to be implemented faithfully. All other terms may be implemented by their canonical equivalent.

d. $\Sigma_{O}=\left(S_{O}, F_{V}\right)$ for some $S_{O} \subseteq S_{V}$, i.e., if $s \in S_{O}$ then all constructor functions for terms of sort $s$ are available, hence $T_{s}=T_{s, O}$ (case b), and, if $s \notin \mathrm{S}_{O}$ then no constructor function for terms of sort $s$ qualifies as outermost function in $T_{O}$, hence $T_{s, O}=\varnothing$ (case a).

This case states that for $s$-observability $\sim_{o}$ is a congruence for terms of any sort $s^{\prime} \in S_{V}$, including $s$ itself. Hence it is a rephrasing of the well-known fact that observability through a sort conserves the congruence (see [MO85]).

Generally $\sim_{o}$ will be a congruence. If that is the case it is usually written as $=_{O}$ in the sequel. Similarly $\sim_{\mathrm{E}, \Sigma}$ becomes $=_{\mathrm{E}, \Sigma}$.

\subsection{Definition (Algebraic Implementation)}

The following definition represents the central notion in this section, namely the notion of implementation for an algebraic specification relative to an observing signature. Intuitively, two specifications are algebraic implementations of each other when they have the same congruence on the observable terms. This is inherently an almost symmetric notion: if a small specification implements part of a large one then the large specification implements the same part of the small one (and more, but that is redundant) if the set of observable terms is the same. We provide the following definition:

- Let $\left(\Sigma_{V}, \Sigma_{H}, \mathrm{E}\right)$ and $\left(\Sigma_{V}^{\prime}, \Sigma_{H}^{\prime}, \mathrm{E}^{\prime}\right)$ be algebraic specifications and $\Sigma_{O}$ be a signature such that $\Sigma_{O} \subseteq \Sigma_{Y} \cap \Sigma_{V}^{\prime}$.

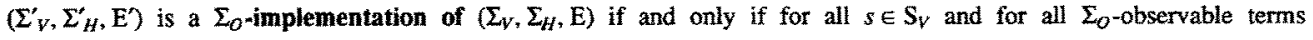
$t, t^{\prime} \in T_{s}\left(\Sigma_{V}\right)$ :

$$
t-E_{E, \Sigma_{0}} t^{\prime} \Leftrightarrow t \sim_{E_{1}^{\prime}, \Sigma_{0}} t^{\prime}
$$

\subsubsection{Some facts about algebraic implementations}

a. If $\Sigma_{O} \subseteq \Sigma_{V}$ then an algebraic specification $\left(\Sigma_{V}, \Sigma_{H}, \mathrm{E}\right)$ is a $\Sigma_{O}$-implementation of itself. As an even more trivial special case $\left(\Sigma_{V}, \Sigma_{\mu}, \mathrm{E}\right)$ is a $\Sigma_{y}$-implementation of itself.

b. If $\left(\Sigma_{V}^{\prime}, \Sigma_{H}^{\prime}, E^{\prime}\right)$ is a $\Sigma_{O}$-implementation of $\left(\Sigma_{V}, \Sigma_{H,}, \mathrm{E}\right)$ and $\Sigma_{O}^{\prime} \subseteq \Sigma_{O}$ then $\left(\Sigma_{V}^{\prime}, \Sigma_{H}^{\prime}, E^{\prime}\right)$ is also a $\Sigma_{O}^{\prime}$-implementation of $\left(\Sigma_{V}, \Sigma_{H}, \mathrm{E}\right)$.

c. $\Sigma_{O}$-implementation is a symmetric relation on the class of algebraic specifications with the same set of $\Sigma_{O}$-observable terms.

d. $\Sigma_{O}$-implementation is also a transitive relation under the conditions of case $c$.

While the facts above provide some idea about the usefulness of the definition two important properties have to be proved. Of course we want to conserve the property in initial algebra semantics that the hidden signature and the set of equations may be changed as long as this does not affect the congruence on the visible signature. This is asserted in lemma 3.2.12.

Next, in the central theorem a functionally oriented criterion is given for an algebraic implementation. This serves as a 
starting point for section 5 , in which a notion of funcaional implementation will be given.

\subsubsection{Initial Algebra Implementation Lemma}

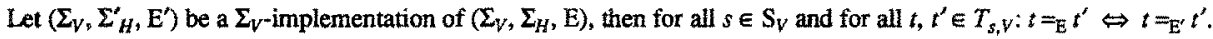
Proof follows from the fact that all terms in $T_{V}$ are $\Sigma_{y}$-observable, and corollary 3.2 .5 (omitted).

\subsubsection{Algebraic Implementation Theorem}

Let $\left(\Sigma_{V}, \Sigma_{H}, \mathrm{E}\right)$ and $\left(\Sigma_{V}^{\prime}, \Sigma_{H}^{\prime}, \mathrm{E}^{\prime}\right)$ be algebraic specifications and $\Sigma_{O} \subseteq \Sigma_{V} \cap \Sigma_{V}^{\prime}$.

If for all $f \in F_{O}, f: s_{1} \times \cdots \times s_{k} \rightarrow s_{0}$, with $s_{0} \in S_{O}$, for all $t \in T_{s_{0}}\left(\Sigma_{V}\right)$ and for all $\left(u_{1}, \cdots, u_{k}\right) \in\left(T\left(\Sigma_{V}\right)\right)^{k}$ $f\left(u_{i}, \cdots, u_{k}\right)={ }_{\mathrm{E}} t \Leftrightarrow f\left(u_{1}, \cdots, u_{k}\right)=\mathrm{E}^{\prime} t$ holds, then $\left(\Sigma_{Y}^{\prime}, \Sigma_{H}^{\prime}, \mathrm{E}^{\prime}\right)$ is a $\Sigma_{O}$-implementation of $\left(\Sigma_{y}, \Sigma_{H}, \mathrm{E}\right)$.

Proof by unfolding all $\Sigma_{O}$-observable terms in $T\left(\Sigma_{V}\right)$ (omitted).

Note: this theorem is sufficiently strong to describe the behaviour of a function up to the congruence defined by $\sim E, \Sigma_{o}$, if such a congruence exists. An example of the use of the theorem is given in the next section. A more restrictive definition of imple mentation, strong enough to describe functional implementation, is given in section 5 .

\section{AN EXAMPLE: TABLES}

In this section, two definitions of elementary data structures for objects of arbitrary sort ELEM are given. Both data structures support storage and retrieval with elements of an arbitrary sort $K E Y$ with equality function eq as selection criterion.

The first specification describes a sort TABLE. An element of this sort is a list of all entries with corresponding keys in the data structure. This data structure can be searched from the last entry to the first, in linear time. The second specification uses a hidden sort TREE to implement the same data in a search-tree. This is possible when we have a total ordering on sort KEY. In the example function It, combined with eq, provides such an ordering.

The specifications below are parameterized with sorts KEY and ELEM at the specification level. To guide the intuition, one should think of two (perhaps equal) sorts which are already well-known, e.g. CHAR for ELEM and INT for KEX. ASF does not provide a semantics for unbound parameter sorts, since there is no mechanism to force restrictions on the actual parameter (iike the total ordering in the example). In the paper restrictions are given in the commentary, so it will be clear what the semantics should be.

The remainder of this section is devoted to a proof sketch of the fact that the modules Tables and Tables-as-trees are algebraic implementations of each other when one observes through the retrieve function lookup only, in other words, with respect to lookup-equality. So we take for the observing signature (\{BOOL, ELEM, KEY, TABLE], [100kup]), or ([ELEM), (lookup]), since ELEM is the range of lookup.

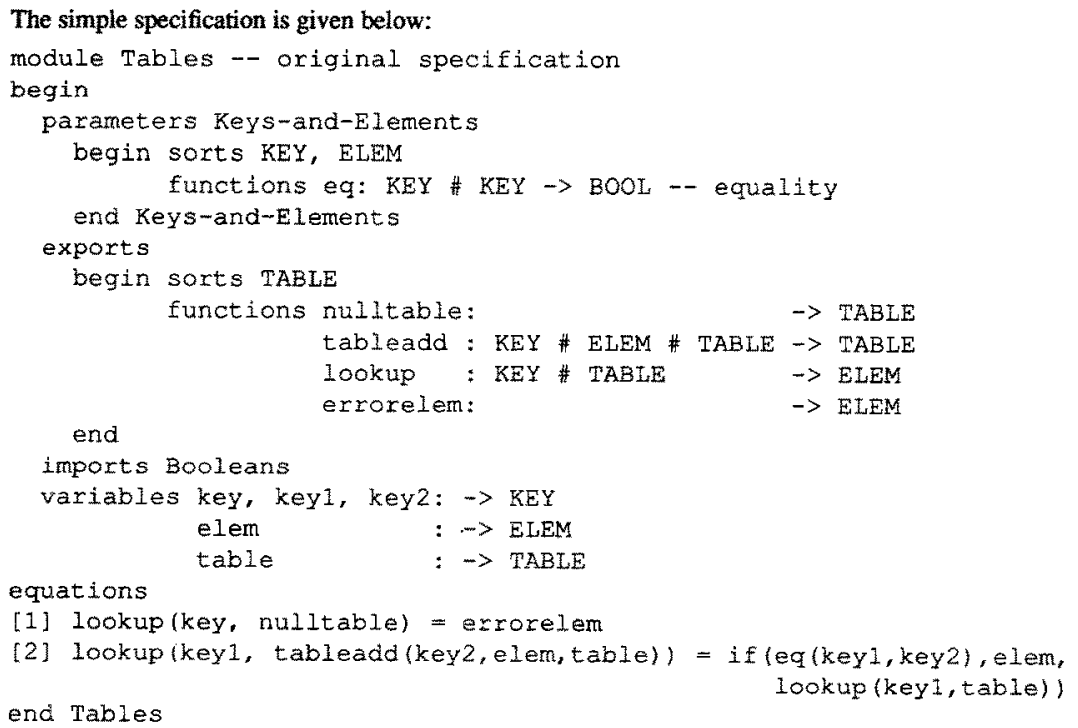

This specification speaks for itself. Function tableadd is underspecified and hence the problems indicated in section 3.1 can arise. To avoid them we restrict the set of observing terms to those with function $100 \mathrm{kup}$ as outermost symbol. Hence an implementor of this module can concentrate on the correct implementation of lookup.

To get an efficient implementation of lookup more detailed information about sort KEX is needed. If KEY is a small set 
something similar to a bounded array is feasible. If a hash function could be defined, a hash table might be used as implementation. Each of these structures can be algebraically specified as hidden structure, thus providing an algebraic specification which upon implementation gives an equivalent, but more efficient, implementation of $100 \mathrm{kup}$.

For this example it is assumed that a total ordering can be defined on the set $\mathrm{KEY}$ with the functions eq and it (lowerthan). The total ordering allows the definition of a binary search-tree. This is done in module Tables-as-trees below:

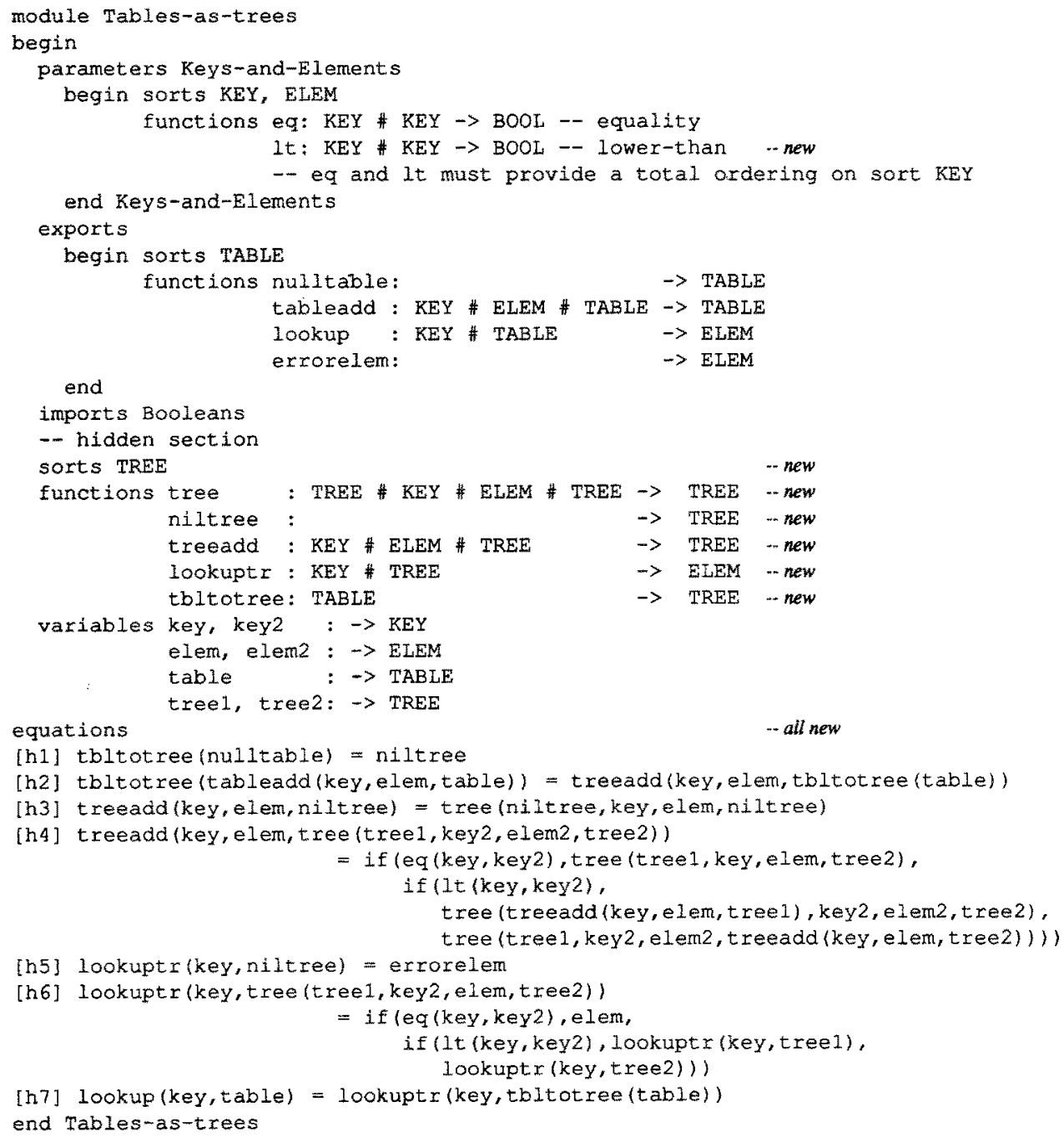

Note that all equations contain hidden sorts. Equation $h 7$ defines lookup in terms of lookupt $r$, the retrieval function on trees, itself defined in $\mathrm{h} 5$ and $\mathrm{h} 6$. Equations $\mathrm{h} 1$ through $\mathrm{h} 4$ define the build-up of a tree from a table.

It is possible to declare all hidden sorts and functions visible rather than hidden. The effect would be that module Tables-as-trees would still be an implementation with respect to lookup-observability of module Tables, but not the other way around. The reason for the latter is the existence of observable terms containing constructor functions for TREE in module Tables-as-trees, terms which are not existent in module tables.

The following proof sketch first defines a well-formedness predicate searchtree for terms of sort TREE, since not all constructible terms are search-trees. Then it is proved that the predicate searchtree is invariant over the insertion function treeadd, and that the retrieval function lookupt $r$ is well-defined for single additions to a tree which satisfies this predicate. Finally the equivalence between the two specifications is proved with induction on the number of insertions. 


\subsection{Definition (well-formedness of searchtrees)}

The predicate searchtree $(t)$ for a term $t$ of sort TREE describes the well-formedness of a tree as search-tree. It can be used in to derive properties about the behaviour of the data structure generated by function treeadd. This hoids in particular for the behaviour observed through function lookupt $r$, which is needed to derive the behaviour of function lookup. The predicate is defined as follows (with $t 1, t 2$ of sort TREE, j, $k, 1$ of sort KEY, and e of sort ENTRY):

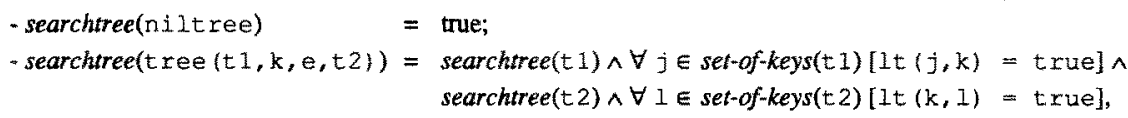

\subsection{Well-formedness lemma for trees}

Let $k, k^{\prime}$ be of sort KEY, e, e' be of sort ENTRY, and t. be of sort TREE, then we can formulate the following

Lemma:

$$
\begin{aligned}
\text { a. searchtree }(t) \rightarrow & \text { searchtree }\left(\text { treeadd }\left(k^{\prime}, e^{\prime}, t\right)\right) . \\
b . \operatorname{searchtree}(t) \rightarrow & {\left[e q\left(k, k^{\prime}\right)=t r u e \rightarrow 100 k u p t r\left(k, t r e e a d d\left(k^{\prime}, e^{\prime}, t\right)\right)=e^{\prime}\right] \wedge } \\
& {\left[e q\left(k, k^{\prime}\right)=f a l s e \rightarrow \operatorname{lookuptr}\left(k, t r e e a d d\left(k^{\prime}, e^{\prime}, t\right)\right)=100 k u p t r(k, t)\right] . }
\end{aligned}
$$

Proof by induction on the number of nodes in the tree (omitted).

\subsection{Proof of lookup-equality}

Lookup-equality can now be proved by induction with respect to the number of insertions using function tableadd. The well-formedness predicate searchtree makes the proof straightforward. If the equivalence defined by the equations from module Tables is called $=_{T b}$ and from module Tables-as-trees $=_{T r}$, then (Theorem 3.2.13) it is sufficient to prove for all pairs $(k, t) \in T_{\mathrm{KEY}, V} \times T_{\text {TABLE, } V}$ and for all terms $e \in T_{\mathrm{ELEM}, V}$

$$
\text { lookup }(k, t)={ }_{T b} \text { e } \Leftrightarrow 100 \mathrm{kup}(k, t)={ }_{T r} \text { e. }
$$

The proof (subsequently omitted) proceeds by assuming that e does not contain the function lookup. Next, it can be extended to general terms $e \in T_{\mathrm{ELEM}, V}$ by replacing such terms with equivalent terms not containing function $100 \mathrm{kup}$.

\section{FUNCTIONAL IMPLEMENTATION}

\subsection{The functional view}

The implementation Theorem (3.2.13 in section 3.2) gives an algebraically clean criterion for implementation. However, it is not sufficient as a tool to fix implementations of functions in the classical sense: a function has a certain result value for every combination of input values. Of course the result value should depend on the input values, but it should not depend on the implementation.

The violation of this property is shown by taking $\Sigma_{V}=(\{s, t),\{a, b, p, q, f\})$ with $a, b \in s, p, q \in t$ and $f: s \rightarrow t, \Sigma_{O}=(\{s, t)$, $\{a, b, \mathrm{f}]), \Sigma_{H}=\varnothing, \mathrm{E}=[\mathrm{f}(a)=p, \mathrm{f}(b)=p]$, and $\mathrm{E}^{\prime}=[\mathrm{f}(a)=q, \mathrm{f}(b)=q]$. The $\Sigma_{0}$-observable terms in $T_{Y}$ are $a, b, \mathrm{f}(a)$ and $\mathrm{f}(b)$. Obviousily $\mathrm{f}(a)={ }_{\mathrm{E}, \Sigma_{0}} \mathrm{f}(b)$ and $\mathrm{f}(a)==_{\mathrm{E}^{\prime}, \Sigma_{o}} \mathrm{f}(b)$. Hence $\left(\Sigma_{V}, \varnothing, \mathrm{E}\right)$ and $\left(\Sigma_{V}, \varnothing, \mathrm{E}^{\prime}\right)$ are $\Sigma_{\sigma}$-implementations of each other. However, $f$ clearly has different result values.

Additional restrictions are needed to be able to view a term in $T_{O}$ as a function (the header function) defined on tuples in $T_{V}$ and with range $T_{V}$. In initial algebra semantics the 'result' is the congruence class defined by the set of equations $E$. Hence any term in the congruence class will do, since it fixes (for specific $\mathrm{E}$ and $\Sigma_{V}$ ) the class. So we need a canonical form, which is a representative for every congruence class. In a confluent and terminating term rewriting system this canonical form is called 'normal form', and it is defined by the system itself.

The following three sets of terms within $T_{Y}$ are induced by $\Sigma_{O}$ :

- the directly $\Sigma_{0}$-observable terms,

- the indirectly $\Sigma_{O}$-observable terms, and

- the terms reachable from $T_{o}$, i.e., terms not necessarily in $T_{o}$ but in the congruence class of some term in $T_{O}$.

Note that the last two sets may overlap. The input values for functions in $F_{O}$ with range in $S_{O}$ form a subset of the union of the first two sets. Any element of the first and the third sets could be in the range of a function in $\mathrm{F}_{O}$.

The directly $\Sigma_{O}$-observable terms do not necessarily contain a desired result value. For example, a specification of stringof-characters might contain a function length from strings to integers. The set of length-observable terms contains the length function applied to various strings, but it does not contain the integers, which is clearly the desired set of result values.

In the subsection below this idea is formalized for a specific observing function. The function has input terms, which should be well-typed, and an output term, depending on the input terms and the set of equations, which must be in a certain set of 
canonical terms. There is an obvious link with the theory of abstract data types (cf. Jones [Jon80]) here. The well-typedness of the input terms serves as precondition and the equations and a characterization of the set of canonical terms serve as postcondition.

In general one has more than one observing function, so some preliminary work has to be done to allow a decomposition of the observing set of functions into singletons.

\subsection{A theory of functional implementation}

\subsubsection{Definitions (input-, reachable and canonical terms)}

Let $\left(\Sigma_{V}, \Sigma_{H}, E\right)$ be an algebraic specification and $\Sigma_{o} \subseteq \Sigma_{V}$. Then

a. the set $I\left(\Sigma_{O}, \Sigma_{V}\right)$ of $\Sigma_{O}$-input terms over $\Sigma_{V}$ is defined as:

$$
I\left(\Sigma_{O}, \Sigma_{V}\right)=\left\{t \in T\left(\Sigma_{V}\right) \mid \exists f \in \mathrm{F}_{O} f: s_{1} \times \cdots \times s_{k} \rightarrow s, s \in \mathrm{S}_{O} \exists i \leq k t \in T_{s_{i}}\left(\Sigma_{V}\right)\right\}
$$

b. the set $R\left(\Sigma_{O}, \Sigma_{V}\right)$ of $\Sigma_{O}$-reachable terms over $\Sigma_{V}$ is defined as: $R\left(\Sigma_{O}, \Sigma_{V}\right)=\left\{t \in T\left(\Sigma_{V}\right) \mid \exists t^{\prime} \in T\left(\Sigma_{O}, \Sigma_{V}\right) t=_{E} t^{\prime}\right\}$.

Note that terms containing hidden functions and sorts are not considered reachable.

c. A set $C\left(\Sigma_{O}, \Sigma_{V}\right) \subseteq R\left(\Sigma_{O}, \Sigma_{V}\right)$ is a set of canonical terms if and only if $\forall t, t^{\prime} \in C\left(\Sigma_{O}, \Sigma_{V}\right)\left[t=t^{\prime} \rightarrow t=t^{\prime}\right]$.

d. A set of canonical terms $C\left(\Sigma_{O}, \Sigma_{V}\right)$ is complete if and only if $\forall t \in T\left(\Sigma_{O}, \Sigma_{V}\right) \exists t^{\prime} \in C\left(\Sigma_{O}, \Sigma_{V}\right) t={ }_{E} t^{\prime}$.

e. A reduction to canonical terms $\rightarrow C\left(\Sigma_{0}, \Sigma_{n}\right.$ ) (abbreviated $\rightarrow C$ or even $\rightarrow$ ) is defined as follows:

$$
t \rightarrow C\left(\Sigma_{0}, \Sigma_{V}\right) t^{\prime} \Leftrightarrow t \in R\left(\Sigma_{O}, \Sigma_{V}\right) \wedge t^{\prime} \in C\left(\Sigma_{O}, \Sigma_{V}\right) \wedge t==_{\mathrm{E}} t^{\prime}
$$

f. Analogous to the definitions of $T_{O}$ and $T_{s}\left(\Sigma_{O}, \Sigma_{Y}\right)$ the following shorthand conventions are adopted:

$$
\begin{array}{lll}
I_{O}=I\left(\Sigma_{O}, \Sigma_{V}\right), & R_{O}=R\left(\Sigma_{O}, \Sigma_{V}\right), & C_{O}=C\left(\Sigma_{O}, \Sigma_{V}\right), \\
I_{s, O}=I_{s}\left(\Sigma_{O}, \Sigma_{V}\right)=I\left(\Sigma_{O}, \Sigma_{V}\right) \cap T_{s}\left(\Sigma_{V}\right), & R_{s, O}=R_{s}\left(\Sigma_{O}, \Sigma_{V}\right)=R\left(\Sigma_{O}, \Sigma_{V}\right) \cap T_{s}\left(\Sigma_{V}\right), & C_{s, O}=C_{s}\left(\Sigma_{O}, \Sigma_{V}\right)=C\left(\Sigma_{O}, \Sigma_{V}\right) \cap T_{s}\left(\Sigma_{V}\right) .
\end{array}
$$

\subsubsection{Some facts}

a. $R\left(\Sigma_{0}, \Sigma_{V}\right) \supseteq T\left(\Sigma_{0}, \Sigma_{V}\right)$.

b. Every term in $I\left(\Sigma_{O}, \Sigma_{V}\right)$ is $\Sigma_{O}$ observable.

c. The converse of fact b does not hold, i.e., not every $\Sigma_{o}$-observable term is a $\Sigma_{0}$-input term.

\subsubsection{Lemma}

Let $C\left(\Sigma_{O}, \Sigma_{Y}\right)$ be a complete set of canonical terms. The terms in $C\left(\Sigma_{O}, \Sigma_{Y}\right)$ with the (adapted) functions on $R\left(\Sigma_{O}, \Sigma_{Y}\right)$ form a canonical term algebra if the operation of these functions on $R\left(\Sigma_{O}, \Sigma_{V}\right)$ is testricted to reach $C\left(\Sigma_{O}, \Sigma_{V}\right)$ by application of the reduction to canonical terms $\rightarrow C$ after the normal application of the function in $R\left(\Sigma_{O}, \Sigma_{V}\right)$. Then $C\left(\Sigma_{O}, \Sigma_{V}\right)$ as a term algebra is isomorphic to $R\left(\Sigma_{O}, \Sigma_{V}\right) /=\mathrm{E}$.

Proof from the definition of $R\left(\Sigma_{O}, \Sigma_{V}\right)$ and $C\left(\Sigma_{O}, \Sigma_{V}\right)$ (omitted).

\subsubsection{Functional decomposition of a reduction to canonical terms}

Next we want to pursue a 'divide and conquer' strategy to provide an implementation of a reduction to canonical terms $\rightarrow$ C. The decomposition chosen is made on the typed head symbol (the "function") of the term to be reduced. This allows for a separate implementation for each function. The total implementation of $\rightarrow c$ can be constructed from the union of these separate implementations.

It should be noted that a reduction to canonical terms $\rightarrow \mathcal{C}$ as a total map from $R_{O}$ to $C_{O}$ is fixed by $C_{O}$ and the congruence $t={ }_{\mathrm{E}}$. This follows from the definition of $C_{O}$, since for every term in $R_{O}$ exactly one term in $C_{O}$ is in the same congruence class. So it is possible to define the map $\rightarrow c$ as a union of partial maps to the set of canonical terms.

It is also possible to define a complete set of canonical terms implicitly by defining a (possibly partial) map $\rightarrow$ from $T_{O}$ to $R_{O}$ for which the following holds:

$$
\begin{aligned}
& \text { 1) } \forall t \in T_{O}, t^{\prime} \in R_{O}\left[t \rightarrow t^{\prime} \Leftrightarrow t=t_{\mathrm{E}} t^{\prime}\right], \\
& \text { 2) } \forall t \in T_{O} \operatorname{card}\left(\left\{t^{\prime} \in R_{O} \mid \exists t^{\prime \prime} \in T_{O}\left[t=\mathrm{E} t^{\prime \prime} \wedge t^{\prime \prime} \rightarrow t^{\prime}\right]\right\}\right)=1 .
\end{aligned}
$$

It can easily be seen that the range of $\rightarrow$ is a complete set of canonical terms. So a reduction to canonical terms can be described by its behaviour on terms in $T_{O}$. A well-known example of such an implicit definition is the set of normal forms defined by a confluent and terminating term rewriting system.

\subsection{Definitions (functional implementation)}

a. Let $\rightarrow c$ be a reduction to canonical terms and let $\Sigma \subseteq \Sigma_{O}$. Then $\rightarrow \Sigma, C$ is defined as the restriction of $\rightarrow c$ to the domain $T\left(\Sigma, \Sigma_{V}\right)$ 
b. Let $\left(\Sigma_{V}, \Sigma_{H}, \mathrm{E}\right)$ be an algebraic specification, $\Sigma_{O}$ be a signature such that $\Sigma_{O} \subseteq \Sigma_{V}$, and $C_{O}$ be a complete set of canonical terms. Then a map $\rightarrow$ is a functional implementation if and only if $\forall t \in T_{O}, t^{\prime} \in C_{O} \quad\left[t \rightarrow t^{\prime} \leftrightarrow t=\mathrm{E} t^{\prime}\right]$.

\subsubsection{Some facts about functional implementations}

a. Let $\left(\Sigma_{V}, \Sigma_{H}, \mathrm{E}\right)$ be an algebraic specification, $\Sigma_{O} \subseteq \Sigma_{V}, C_{O}$ a set of canonical terms, and ${ }^{\prime} C^{1} T_{0}$ the restriction of reduction to canonical terms $\rightarrow{ }_{C}$ to domain $T_{O}$. Then $\left.\rightarrow C{ }^{1} T_{b}=\bigcup_{f \in F_{o}} \rightarrow\left(s_{b}, U\right\}\right), C$ holds.

Hence $\rightarrow c c^{i} T_{0}$ (and according to section 5.2 .4 thus by extension $\rightarrow_{C}$ ) can be defined for each function in $\Sigma_{O}$ separately.

b. Let $\left(\Sigma_{V}, \Sigma_{H}, \mathrm{E}\right)$ and $\left(\Sigma_{V}^{\prime}, \Sigma_{H}^{\prime}, \mathrm{E}^{\prime}\right)$ be $\Sigma_{O}$-implementations of each other (so $\Sigma_{O} \subseteq \Sigma_{V} \cap \Sigma_{V}^{\prime}$ ). Then a functional implementation $\rightarrow C \subseteq T_{O} \times C_{O}$ of $\left(\Sigma_{V}^{\prime}, \Sigma_{H}^{\prime}, \mathrm{E}^{\prime}\right)$ is also a functional implementation of $\left(\Sigma_{V}, \Sigma_{H}, \mathrm{E}\right)$ if for all $t \in T_{O}, t^{\prime} \in C_{O} t=\mathrm{E}^{\prime} \leftrightarrow t=\mathrm{E}^{\prime} t^{\prime}$ holds.

c. If two algebraic specifications are $\Sigma_{O}$-implementations of each other and both have a functional implementation then these implementations are isomorphic.

\subsubsection{Concrete representation}

Eventually, we want to convert an algebraic specification into a working computer program. For this a representation function 1 from the set of input terms $I_{O}$ to the concrete representation of input terms is needed to be able to execute implemented functions. When confusion arises the restriction of $\mathfrak{l}$ to the domain $I_{s, O}$ will be written as $\mathfrak{l}_{s}$. Additionally, a set of retrieval functions $\rho_{s}$ from concrete representations of output terms to the set of canonical terms $C_{s, O}$ is needed.

This is formalized in the following definitions:

Let I be a set of data types for a programming language $L$. I is an implementation in $L$ of $I_{O}$ and $R_{O}$ if there is a total function $\mathrm{t}: I_{O} \rightarrow I$ (the implementation function) and a set of (partial) functions $\left\{\rho_{s}: \mathrm{I} \rightarrow I_{s, O} \cup R_{s, o} \mid s \in \mathrm{S}_{O}\right\}$ (the retrieval functions) such that $p_{s}(u(t))=0 t$ for all $t \in I_{s, O}$.

Generally, if $I_{s, O}$ is not empty then $t\left(I_{s, o}\right)$ will correspond to a subset of a data type in $L$. It could very well happen that two different input types are implemented by the same data type, so differently named retrieval functions are needed for every sort. Only one name $(l)$ is needed for the implementation function, since the sort of the argument provides type information.

\subsubsection{Implementation theorem}

Let $\left(\Sigma_{V}, \Sigma_{H}, E\right)$ be an algebraic specification, $\Sigma_{O} \subseteq \Sigma_{V}$, and $C_{O}$ a set of canonical terms. Let I be an implementation in a programming language $L$ of $I_{O}$ and $R_{O}$ with implementation function 1 and retrieval functions $\left\{\rho_{s} \mid s \in \mathrm{S}_{O}\right\}$, and $\mathrm{S}\left(x_{1}, \cdots, x_{n}\right)$, storing its result in $c$, a program operating on 1 . Then the statement $S\left(x_{1}, \cdots, x_{n}\right)$, describes a functional implementation $\rightarrow\left(S_{o}, U l\right), C$ for $f: s_{1} \times \cdots \times s_{n} \rightarrow s, s \in S_{O}$, if the following holds:

$$
\begin{aligned}
& \left\{c_{1} \in s_{1} \wedge \cdots \wedge c_{n} \in s_{n} \wedge k_{1}=\mathbf{t}\left(c_{1}\right) \wedge \cdots \wedge k_{n}=\mathbf{l}\left(c_{n}\right)\right\} \\
& \mathrm{S}\left(k_{1}, \ldots, k_{n}\right) \\
& \left\{\rho_{s}(c) \in C_{s, o} \wedge f\left(c_{1}, \ldots, c_{n}\right)=\mathrm{E} \rho_{s}(c)\right\} .
\end{aligned}
$$

Proof proceeds by looking at the function defined by $S$ (omitted).

\subsubsection{Decidability of the conditions}

It is a pleasant property of Theorem 5.2 .8 that in practice satisfaction of the precondition can be computed if the implementation function $l$ can be computed. Since the terms in $I_{O}$ are typed, a typechecking algorithm provides the statements on membership of the input terms. Generally there are no extra restrictions to ensure computability, since obviously the implementation has to be computed anyway.

The decidability of the postcondition depends on the computability of the retrieval function $\rho_{s}$, the decidability of the check on membership of the set of canonical terms $C_{O}$, and the decidability of the congruence $=_{\mathrm{E}}$. The first condition is necessarily fulfilled for the same reasons as the computability of the implementation function. The second depends on the definition of $C_{O}$, which will allow computation in practical cases (who wants a canonical form wild enough to be unrecognizable as such?). The decidability of $\mathrm{E}_{\mathrm{E}}$ is not ensured in general. So a separate proof may be needed. Of course, for many classes this congruence is decidable. For specifications where the congruence is undecidable, e.g. an algebraic specification of a programming language, an implementation will provide at least a partial decision procedure, even when it cannot be completed.

\section{AN EXAMPLE: TABLES REVISTED}

To illustrate the use of Theorem 5.2.8 an implementation of module Tables in an imperative language is given. Though the implementation again uses trees there is an important difference with the algebraic implementation in section 4 in the sense that rectrsion is eliminated.

The language Pascal (described in (JW78]) is chosen for the imperative implementation. This choice is motivated by its 
availability and by its convenient type system. Of course, any other imperative language would serve as well. It should be noted that a functional implementation is very well possible, even in Pascal, but we want to illustrate the possibility to give a correct implementation in a non-functional way.

Generally it is easier to derive a functional program from an ASF-specification, since writing an algebraic specification has strong similarities to functional programming. The specification of Tables-as-trees, for instance, is easily converted into a functional program for lookup. Thus a functional implementation has the advantage of being easily derived from the specification, and also of being faster in general than a term rewriting implementation.

The first step in the implementation is the choice of a data structure. This is provided for by the data type declarations pointer $=\uparrow$ tree, and tree as a record with fields $l, r$ (pointers to the left and right subtrees), $k$ of type key, and $e$ of type elem. In a concrete program it is necessary to bind the sorts key and elem. One could take, for instance, integers and characters; the only prerequisite is that an ordering must be established on the keys.

The values niltree and errore lern require different treatment in Pascal. For the first we can use the standard notion nil, the second has to be declared as variable and set to some unused value. The auxiliary functions on key pose no problems with the current choice, since integers are already ordered. For familiarity reasons the function names $e q$ and $l t$ are retained instead of the operators $=$ and $<$.

Next the implementation function 1 must be defined. The domain of $t$ is $T_{K E Y, V} \cup T_{\mathrm{ELEH}, V} \cup T_{\mathrm{TABLE}, V}$ and its range is the union of the data types key, elem and tree (or rather pointer to tree) already indicated above. Since a specification of the terms of type ELEM and KEY has not been given in section 3 an identification with elem and key is assumed, so 1 is 'defined' backwards by $t(t)=t$ for $t \in T_{\mathrm{XEY}, Y} \cup T_{\mathrm{ELEM}, y}$. Hence also $\rho_{\mathrm{ELEM}}(t)=t$, the only retrieval function needed for the example. For $t \in T_{\text {table, } V}, l$ can be defined by $1($ nulltable $)=n i l$ and $u($ tableadd $(k e y$, elem, table $)$ ) $=p t r$ when treeadd (l(key), 1 (elem),ptr) is executed with $p t r=1(t a b l e)$.

This definition uses procedure treeadd defined below. It should be noted that function 1 restricted to terms of type TABLE plays the same role as function tbltotree in section 4. Evidently, procedure treadd below and function treeadd in section 4 are closely related also. A procedure with a variable parameter is a common way to handle data structures in a language like Pascal. A function definition would have the advantage of a more elegant definition of function 1 , but the definition below shows that other programming styles can be handled too.

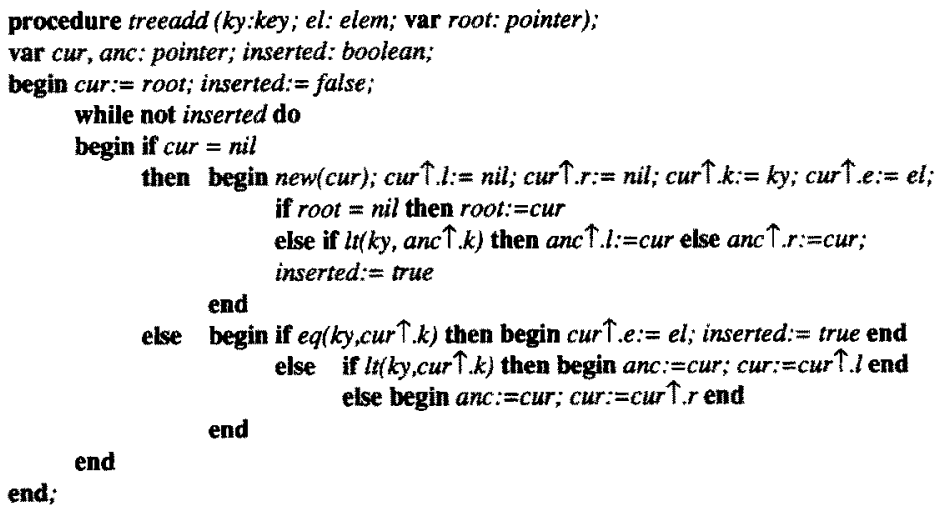

The proof of correctness of this implementation closely resembles the proof sketch in section 4 . Following this lead we provide two well-formedness functions on structures of type pointer (to tree), again called searchtree and set-of-keys. They are defined in the obvious way. This allows us to state the well-formedness of implemented terms by providing the following parallel to Lemma 4.2.a:

6.1. Second well-formedness lemma for trees (part a)

Let $p t r$ be of type pointer and $t \in T_{\text {TABLE, } V}$. Then $p t r=1(t) \rightarrow$ searchtree $(p t r)$.

Proof by induction on the number of nodes in the list (omitted).

Next we provide the function lookuptr:

function lookuptr (ky: key; root: pointer): elem;

var cur: pointer; searched: boolean;

begin cur:= root; searched: $=$ false;

while not searched do

begin if cur $=$ nil 


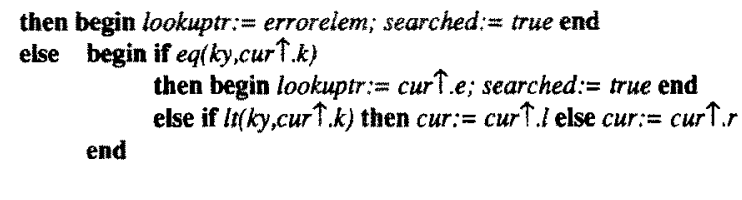

end:

Presently, a lemma similar to lemma $4.2 . b$ can be formulated. It states that lookuptr is well-defined for single additions to a well-formed tree.

\subsection{Second well-formedness lemma for trees (part $b$ )}

Let $k, k^{\prime}$ be of sort KEY, e of sort ENTRY, and $t$ of sort TREE, and let $p t r^{\prime}=u\left(t\right.$ reeadd $\left.\left(k^{\prime}, e, t\right)\right)$. Then

$p t r=\mathfrak{l}(\mathrm{t}) \rightarrow\left[\right.$ eq $\left(\mathrm{l}(\mathrm{k}), \mathrm{t}\left(\mathrm{k}^{\prime}\right)\right)=$ true $\rightarrow$ lookuptr $\left.\left(\mathrm{l}(\mathrm{k}), p t \mathrm{r}^{\prime}\right)=\mathrm{e}\right] \wedge$

$$
\left.\left.\left[e q\left(\mathrm{l}(\mathrm{k}), \mathfrak{l}\left(\mathrm{k}^{\prime}\right)\right]=\text { false } \rightarrow \text { lookuptr( } \mathrm{l}(\mathrm{k}), \mathrm{ptr}\right)=\text { lookuptr } \mathrm{l}(\mathrm{k}), \mathrm{ptr}\right)\right]
$$

Proof directly from the observation that $p t r^{\prime}=l\left(t\right.$ reeadd $\left.\left(k^{\prime}, e, t\right)\right)$ is defined in terms of $p t r=l(t)$ (omitted).

According to Theorem 5.2 .8 it is now sufficient to prove ( $E$ the set of equations from module Tables):

$\{k \in K E Y \wedge t b l \in T A B L E \wedge k y=l(k) \wedge$ root $=\mathfrak{l}(t b l)\}$

elt $:=$ lookuptr $(k y, r o o t)$

$\left(\rho_{\text {ELEM }}(e l t) \in C_{\text {ELEM }_{z} O} \wedge 100 k u p(k, t b I)=\rho_{\mathrm{E}} \rho_{\text {ELEM }}(e l t)\right)$.

This follows immediately from lemma 6.2 , and the definition of $\rho_{\mathrm{ELEH}}$.

\section{CONCLUSIONS}

\subsection{The results}

The paper provides a functionally oriented (black box) approach to the implementation of modular algebraic specifications. The main advantages are listed below.

- It provides a theoretical background for the separate implementation of modules.

- The implementation above is based on the initial behaviour of certain functions, the observing functions. This provides an intuitively clear semantics.

- A correctness criterion for implementations is given in Hoare logic, allowing the application of standard optimization techniques. In algebraic terms this means that functions which are not observing may have more or less final semantics.

- The combination of separate implementation and (hence separate) optimization allows the construction of a library of (possibly optimized) modules.

The loss of the initial algebra semantics might instead be listed as a disadvantage. Terms are only judged different when they have different effects (confusion is allowed) and other invisible terms (junk) may be introduced. On the one hand, precisely these two "undesirable" effects allow the introduction of optimal implementations. On the other hand, they make the seman" tics of a module less clear to the user (i.e., someone writing a module importing the optimized module). This problem is minimized by the fact that the criteria for use of the module, allowing the set of observing terms only, are rather easy.

\subsection{Further research}

Both theoretical and practical extensions of the work in this paper are feasible. The former include:

- Investigation of the significance for import semantics in an algebraic specification formalism with initial algebra semantics, except for observable imports.

- The combination with an implementation as a term rewriting system of the importing module of an observable implementation has to be investigated to allow for automatic translation of modules built around an observable module.

Of more practical nature are:

- The design and implementation of a module library, containing efficient (e.g. built-in) implementations.

- The construction of an implementation of modules on top of the module library, using the normalization (i.e., elimination of imports, renamings and bindings by combining modules) semantics from ASF [BHK87] for the top level modules.

\section{Acknowledgements}

Discussions with P,RH. Hendriks, P. Klint, and especially J. Heering greatly improved the content of this paper. They, J. Rekers, and the referees for ESOP ' 88 made useful comments on one or more earlier versions. 


\section{REFERENCES}

[Bac86] R.C. BACKHOUSE (1986). Program Construction and Verification, Prentice-Hall.

[Bak84] C. BAKER-FINCH (1984). "Acceptable models of algebraic semantics," Australian Computer Science Communications, vol. 6, no. 1, pp. 5 1/10, Proceedings of the Seventh Australian Computer Science Conference, Adelaide, ed. C.J. Barter.

[BHK85] J.A. BERGSTRA, J. HEERING, and P. KLINT (1985). "Algebraic definition of a simple programming language," Report CS-R8504, Centre for Mathematics and Computer Science, Amsterdam.

[BHK86] J.A. BERGSTRA, J. HEERING, and P. KLINT (1986). "Module algebra," Report CS-R8617, Centre for Mathematics and Computer Science, Amsterdam.

[BHK87] J.A. BERGSTRA, J. HEERING, and P. KLINT (1987). "ASF - an algebraic specification formalism," Report CSR8705, Centre for Mathematics and Computer Science, Amsterdam.

[BK86] J.A. BERGSTRA and J.W. KLOP (1986). "Conditional rewrite rules: confluence and termination," Journal of Computer and System Sciences, vol. 32, no. 3, pp. 323-362.

[BT82] J.A. BERGSTRA and J.V. TUCKER (1982). "The completeness of the algebraic specification methods for computable data types," Information and Control, vol. 54, no. 3, pp, 186-200.

[BT83] J.A. BERGSTRA and J.V. TUCKER (1983). "Initial and final algebra semantics for data type specifications: two characterization theorems," SIAM Journal on Computing, vol. 12, no. 2, pp. 366-387.

[BDMW81] M. BROY, W. DOSCH, B. MöLLER, and M. WIRSING (1981). "GOTOs - a study in the algebraic specification of programming languages," in $G I-11$. Jahrestagung, ed. W. Brauer, Informatik-Fachberichte, vol. 50, pp. 109. 121, Springer-Verlag.

[Die86] N.W.P. VAN DIEPEN (1986). "A study in algebraic specification: a language with goto-statements," Report CSR8627, Centre for Mathematics and Computer Science, Amsterdam.

[DE84] K. DROSTEN and H.-D. EHRICH (1984). "Translating algebraic specifications to Prolog programs," InformatikBericht Nr. 84-08, Technische Universität Braunschweig.

[FGJM85] K. FUtATSUGi, J.A. Goguen, J.-P. JouANNAUd and J. MEseguer (1985). "Principles of OBJ2", in Canjerence Record of the Twelfth Annual ACM Symposium on Principles of Programming Languages, pp. 52-66, ACM.

[GM82] J.A. GOGUEN and J. MESEGUER (1982). "Universal realization, persistent interconnection and implementation of abstract modules," in Proceedings 9th International Conference on Automata, Languages and Programming, eds. M. Nielsen \& E.M. Schmidt, Lecture Notes in Computer Science, vol. 140, pp. 265-281, Springer-Verlag.

[GM84] J.A. GOGUEN and J. MESEGUER (1984). "Equality, types, modules, and (why not?) generics for logic programming," Journal of Logic Programming, vol. 2, pp. 179-210.

[GMP83] J.A. GoGUEN, J. MESEGUER and D. PLAISTED (1983). "Programming with parameterized abstract objects in OBJ', in Theory and Practice of Software Technology, eds. D. Ferrari, M. Bolognani \& J.A. Goguen, pp. 163. 193, North-Holland.

[HO80] G. HUET and D.C. OPPEN (1980). "Equations and rewrite rules: a survey," in Formal Language Theory, Perspectives and Open Problems, ed. R.V. Book, pp. 349-405, Academic Press.

[JW78] K. JENSEN and N. WIRTH (1978). Pascal: User Manual and Report (second edition), Springer-Verlag.

[Jon80] C.B. JONES (1980). Software Development: a Rigorous Approach, Prentice-Hall.

[Kam83] S. KAMIN (1983). "Final data types and their specification," ACM Transactions on Programming Languages and Systems, vol. 5 , no. 1, pp. 97-123.

[LS84] J. LOECKX and K. SiEBER (1984). The Foundation of Program Verification, Wiley-Teubner.

[MG85] J. MESEGUER and J.A. GOGUEN (1985). "Initiality, induction, and computability," in Algebraic Methods in Semantics, eds. M. Nivat \& J.C. Reynolds, pp. 459-541, Cambridge University Press.

[ODo85] M.J. O'DONNELL (1985). Equational Logic as a Programming Language, MIT Press.

[ST85] D. SANNELLA and A. TARLECKI (1985). "On observational equivalence and algebraic specification," in Mathematical Foundations of Software Development. Proceedings International Joint Conference on Theory and Practice of Software Development, TAPSOFT' 85 , eds. H. Ehrig, C. Floyd, M. Nivat \& J. Thatcher, Lecture Notes in Computer Science, vol. 185, pp. 308-322, Springer-Verlag.

[Wan79] M. WAND (1979). "Final algebra semantics and data type extensions," Joumal of Computer and System Sciences, vol. 19 , pp. $27-44$. 